\title{
Defibrillation in Models of Cardiac Muscle
}

\author{
ARUN V. HOLDEN \\ Department of Physiology and Centre for Nonlinear Studies, The University, Leeds LS2 9JT, UK
}

(Received I October 1996)

\begin{abstract}
The propagation of electrical activity in cardiac tissue can be modelled by reaction diffusion equations, where a tensor of diffusion coefficients represents anisotropy due to fibre orientation, and excitation is represented by high-order, stiff differential systems. The effects of external electrical stimulation, as in artificial pacemakers, or in defibrillators, requires bidomain models, in which intra- and extracellular currents are treated separately. Simplified approaches are taken to this problem to illustrate two methods of defibrillation: by a single large pulse, that eliminates all propagating activity, and by a series of smaller amplitude perturbations, that drive out re-entrant sources of excitation.
\end{abstract}

Keywords: arrhythmia, cardiac muscle, detibrillation

\section{INTRODUCTION}

The rhythmic beating of the heart is triggered by waves of electrical activity, the propagating cardiac action potential, that are initiated in a specialised, autorhythmic pacemaker region, and spread through the atria and ventricles through the cardiac tissue, and from the atria to the ventricles by specialised conducting pathways. The rate of beating is controlled by the pacemaker rate, and the pattern of activity arises from propagation through the anisotropic, anatomically organised cardiac muscle.

Disturbances in propagation, or abnormal, ectopic pacemaker sites, can give rise to arrhythmias that can range from being unnoticed to lethal. A dangerous class of arrhythmia are the re-entrant arryhthmias, in which the same wave of excitation repeatedly re-invades the same piece of tissue; these re-entrant arrhythmias are high frequency, as the period of the re-entrant wave is less than the normal period of the heartbeat, and underly atrial flutter and monomorphic ventricular tachycardia. If re-entrant waves break down, due to their intrinsic instability, or the effects of anisotropy and the geometry of the heart, spatio-temporal irregularity in the pattern of activation produces fibrillation, in which different parts of the same chamber of the heart are activated at different times. Global coordination of the contraction of the heart is lost and instead of pumping rhythmically the heart writhes and quivers. The circulation is no longer maintained and death can result if the heart is not defibrillated (Zipes and Jalife, 1995).

Here I consider mathematical aspects of defibrillation by applied electrical shocks or perturbations, where by defibrillation I mean eliminating single and multiple re-entrant wave sources in the heart. The aim is to illustrate the mathematical basis, in terms of reaction-diffusion models for excitable media; important practical technical details, such as 


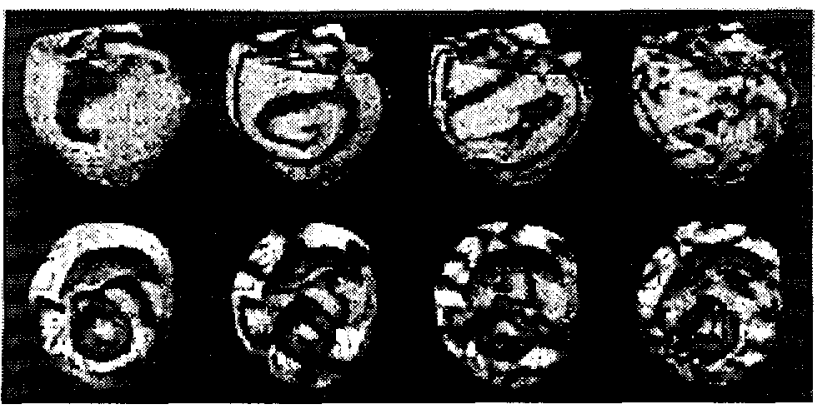

FIGURE 1.1 Frames from a movie illustrating the irregular pattern of propagation in the canine ventricles during fibrillation.
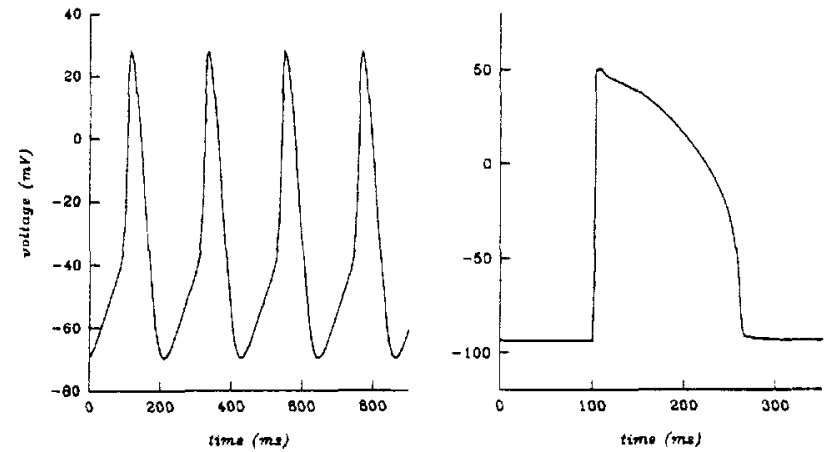

FIGURE 1.2 Simulated action potentials for rabbit sinoatrial node: pacemaker activity as a limit cycle trajectory (left) and an evoked action potential in guinea pig ventricular muscle: solution of a stiff ODE. Oxsoft model equations.

electrode geometry and position, and control algorithms, are omitted.

\section{ODE MODELS OF EXCITATION}

Membrane excitation equations are of the form:

$$
\begin{aligned}
C \partial_{t} u & =f(u, v, w), \\
\partial_{t} v & =g(u, v, w), \\
\partial_{t} w & =h(u, v, w)
\end{aligned}
$$

where $u=u(t)$ is the transmembrane voltage, $C$ is specific membrane capacitance, $f$ is transmembrane current density, vector $v=v(t)$ describes the fast gating variables, and vector $w=w(t)$ comprises slow gating variables and intra- and extracellular ionic concentrations, and $g$ and $h$ describe their kinetics. The variables $u$ and $v$ have comparable characteristic times.

$f$ can be represented by a simple caricature, such as the FitzHugh-Nagumo (FHN) equations, that retain the cubic characteristics of excitability, or by empirical biophysical excitation equations derived from voltage clamp experiments. The phenomenology of excitation in two- and three-dimensional, isotropic and anisotropic excitable media, and in anatomically accurate ventricular wall models, can be explored using FHN models. However, for simulating patterns of propagation in real cardiac tissue, and cardiac arrhythmias and their control, biophysically accurate equations are necessary. Biophysical excitation equations have been obtained for various cardiac tissues; these are high-order, stiff, systems of nonlinear differential equations, and are continually being modified and revised as new experimental observations are incorporated: see chapter 1 of Panfilov and Holden (1996). In this review we use only excitation models from the Oxsoft family, for rabbit sino-atrial node and atrial and guinea-pig ventricular cells (Noble, 1994).

The bifurcation behaviour of these highorder ODEs can be explored numerically using 


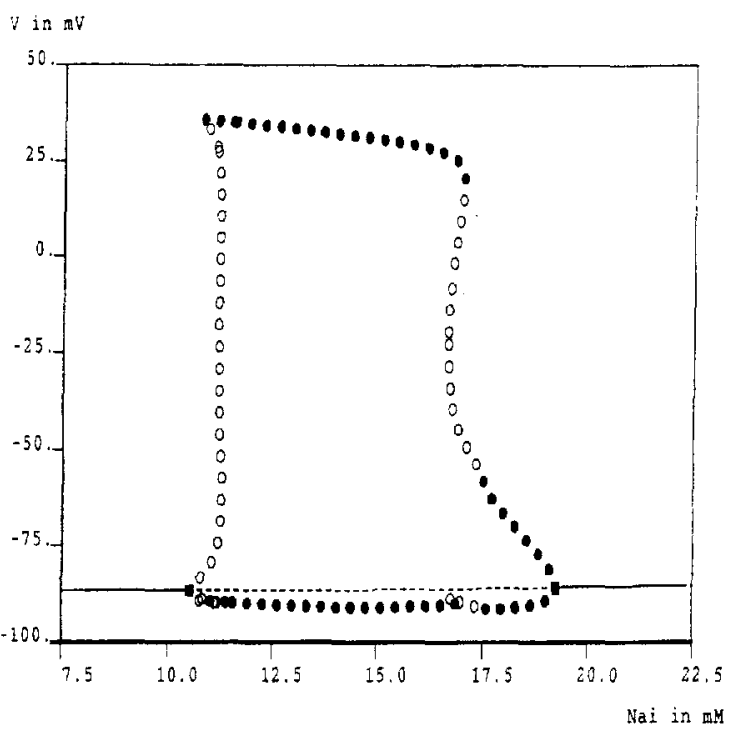

FIGURE 1.3 Equilibrium (solid line: stable; dashed line: unstable) and maxima and minima of stable $(\bullet)$ and unstable (O) periodic solutions for Oxsoft rabbit atrial cell model as $\left[\mathrm{Na}_{i}\right]$ is varied as a bifurcation parameter.

continuation algorithms, and the common behaviour as a parameter is changed is an equilibrium solution losing, and then regaining, its stability at Hopf bifurcations. Thus the cardiac cell models are either excitable, with a stable equilibrium solution, or endogeneously active pacemakers, with a stable limit cycle. Period doubling bifurcations (into bigeminy) have been seen, but patterned and irregular (chaotic) solutions are only found in restricted and unphysiological regions of parameter space.

A simple model of abnormal pacemaker activity induced by ischaemia is to increase $\left[\mathrm{Na}_{1}^{+}\right]$; this simulates the effects of a block of the $\mathrm{Na}^{+}-\mathrm{K}^{+}$ exchange pump. At elevated $\left[\mathrm{Na}_{\mathrm{i}}^{+}\right]$periodic oscillations emerge: Winslow et al. (1993) have shown that a small cluster of such rhythmically active atrial cells can act as an ectopic pacemaker site, producing an arrhythmia.

\section{PDE MODELS OF PROPAGATION}

For a one dimensional excitable medium with a nonlinear current-voltage relation for the ionic current density $I_{\text {ion }}$ (measured in $\mu \mathrm{A} \mathrm{cm}^{-2}$ )

$$
C_{\mathrm{m}} \partial V / \partial t=\partial / \partial x(G \partial V / \partial x)-I_{\mathrm{ion}},
$$

where $G$ is a conductance, with units of $S$. If we divide by $C_{\mathrm{m}}$ we obtain

$$
\partial V / \partial t=\partial / \partial x(D \partial V / \partial x)+I_{\text {ion }} / C_{\mathrm{m}}
$$

where $D$ is a diffusion coefficient with units of $\mathrm{cm}^{2} \mathrm{~ms}^{-1}$. Experimental preparations in cardiac electrophysiology are short compared to the spatial extent of a propagating action potential and so a finite cable, with Neumann boundary conditions, is usually appropriate.

To consider the effects of external stimulation the pattern of current flow in both the intra- and extracellular domains needs to be considered. Let $V_{\text {ex }}$ and $V_{\text {int }}$ be potentials and $D_{\text {ex }}$ and $D_{\text {int }}$ be conductivities in extracellular and intracellular spaces. Assuming Ohmic conductivities, the currents in extracellular and intracellular spaces are given by:

$$
I_{\mathrm{ex}}=-D_{\mathrm{ex}} \frac{\partial V_{\mathrm{ex}}}{\partial x} I_{\mathrm{int}}=-D_{\mathrm{int}} \frac{\partial V_{\mathrm{int}}}{\partial x}
$$

From the conservation law for current flow:

$$
\frac{\partial I_{\mathrm{ex}}}{\partial x}=i_{\mathrm{m}}, \frac{\partial I_{\mathrm{int}}}{\partial x}=-i_{\mathrm{m}},
$$

The membrane current $i_{\mathrm{m}}$ is a sum of ionic and capacitive current, therefore the equations become:

$$
\begin{aligned}
& C_{\mathrm{m}} \frac{\partial V_{\mathrm{m}}}{\partial t}=-\frac{\partial}{\partial x}\left(D_{\mathrm{ex}} \frac{\partial V_{\mathrm{ex}}}{\partial x}\right)+I_{\mathrm{ion}}\left(V_{\mathrm{m}}, g_{\mathrm{i}}\right), \\
& C_{\mathrm{m}} \frac{\partial V_{\mathrm{m}}}{\partial t}=\frac{\partial}{\partial x}\left(D_{\mathrm{int}} \frac{\partial V_{\text {int }}}{\partial x}\right)+I_{\text {ion }}\left(V_{\mathrm{m}}, g_{\mathrm{i}}\right),
\end{aligned}
$$

where $V_{\mathrm{m}}=V_{\text {int }}-V_{\mathrm{ex}}$ is a transmembrane potential. Alternatively, a linear combination gives:

$$
\begin{aligned}
& C_{\mathrm{m}} \frac{\partial V_{\mathrm{m}}}{\partial t}=-\frac{\partial}{\partial x}\left(D_{\mathrm{ex}} \frac{\partial V_{\mathrm{ex}}}{\partial x}\right)+I_{\mathrm{ion}}\left(V_{\mathrm{m}}, g_{\mathrm{i}}\right) \\
& \frac{\partial}{\partial x}\left(D_{\mathrm{ex}} \frac{\partial V_{\mathrm{ex}}}{\partial x}+D_{\mathrm{int}} \frac{\partial V_{\mathrm{int}}}{\partial x}\right)=0
\end{aligned}
$$

These equations form the basis of bidomain models for cardiac tissue. In the one-dimensional case, we 

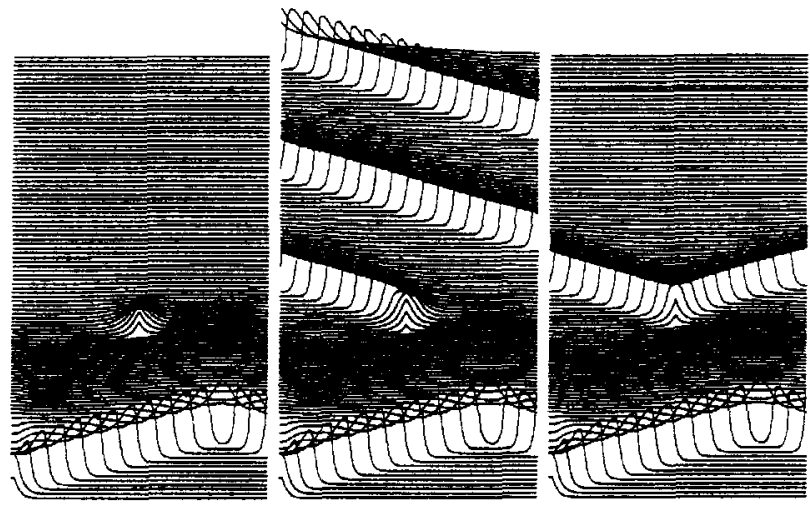

FIGURE 1.4 The response of a homogeneous atrial ring model to a test stimulus applied to the refractory tail of a propagating conditioning wave $\Delta t$ after the conditioning wave front reaches the stimulation site. (Left) $\Delta t=80 \mathrm{~ms}$, no action potential initiated; (Centre) $\Delta t=83 \mathrm{~ms}$, unidirectional block; (Right) $\Delta t=90 \mathrm{~ms}$, a pair of action potential initiated. Membrane potential is displayed against distance.

can always reduce the number of equations from two to one as:

$$
C_{\mathrm{m}} \frac{\partial V_{\mathrm{m}}}{\partial t}=\frac{\partial}{\partial x}\left(\frac{D_{\mathrm{ex}} D_{\mathrm{int}}}{D_{\mathrm{int}}+D_{\mathrm{ex}}} \frac{\partial V_{\mathrm{m}}}{\partial x}\right)+I_{\mathrm{ion}}\left(V_{\mathrm{m}}, g_{\mathrm{i}}\right) .
$$

For two or three dimensional media instead of a simple conductance we have a conductivity tensor, i.e. a matrix which gives the proportionality between voltage gradient and current density.

$$
J_{i}=D_{\mathrm{ij}}, \frac{\partial V}{\partial x_{j}}
$$

(here and throughout the rest of this section we assume the summation convention i.e. sum over doubly repeated indices) where $V$ is potential, $J_{i}$ is a vector of current density, and $D$ is a symmetric tensor accounting for conductivity of the medium, which in two dimensions has the following form:

$$
D=\left(\begin{array}{ll}
D_{11} & D_{12} \\
D_{12} & D_{22}
\end{array}\right)
$$

Following the same procedure as for one dimensional case we obtain:

$$
\begin{aligned}
& C_{\mathrm{m}} \frac{\partial V_{\mathrm{m}}}{\partial t}=\frac{\partial}{\partial x_{i}}\left(D_{i j}^{\mathrm{int}} \frac{\partial V_{\text {int }}}{\partial x_{j}}\right)+I_{\text {ion }}\left(V_{\mathrm{m}}, g_{\mathrm{i}}\right), \\
& \frac{\partial}{\partial x_{i}}\left(D_{i j}^{\mathrm{int}} \frac{\partial V_{\text {int }}}{\partial x_{j}}+D_{i j}^{\mathrm{ex}} \frac{\partial V_{\mathrm{ex}}}{\partial x_{j}}\right)=0
\end{aligned}
$$

where $D_{i j}^{\text {int }}$ and $D_{i j}^{\text {ex }}$ are conductivity tensors for intracellular and extracellular spaces.

A resting one-dimensional medium responds to a brief localised excitation by either a subthreshold disturbance, or by a pair of travelling wave solutions (action potentials). Stimulation in the wake
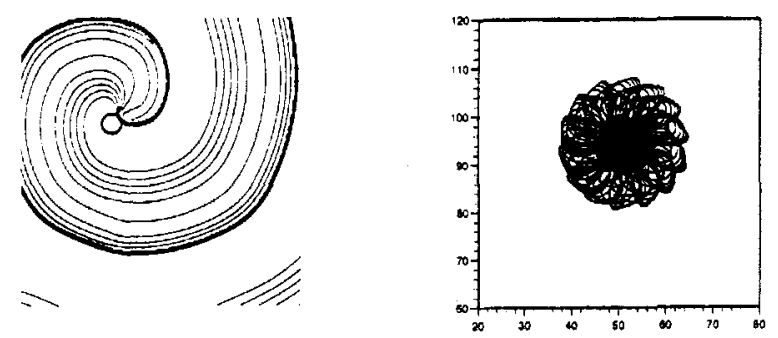

FIGURE 1.5 (Left) Spiral wave solution displayed as voltage contours every $10 \mathrm{mV}$ in a square rabbit atrial tissue model, the spiral rotates around a circular core; (Right) trajectory of tip of spiral wave for first $10 \mathrm{~s}$ of rotation of spiral, aging (due to activity-dependent slow changes in intracellular and extracellular ionic concentrations) changes the pattern of motion from rigid rotation around a small circular core to a biperiodic motion. 
of an action potential, during a vulnerable window, can excite a unidirectional action potential; in a one-dimensional model with a ring topology this provides a simple model for re-entrant excitation, when the same action potential repeatedly re-invades the same piece of tissue. Such re-entry underlies a class of cardiac arrhythmias, and so pharmacological modification of the vulnerablity of cardiac tissue to re-entry can be explored with biophysical excitation models on a ring.

Re-entry in two-dimensional models has the characteristics of a spiral wave, that can rotate rigidly around a circular core, or meander biperiodically. Numerical studies of spiral wave solutions in atrial (Holden and Zhang, 1994) and ventricular (Holden et al., 1996) tissue models also show aging: the pattern of meander changes with time.

We now want to eliminate re-entrant waves by applying external stimulation, and so need to obtain the response of cardiac tissue to externally applied stimulation.

\section{THEORY OF SINGLE SHOCK DEFIBRILLATION}

We begin with a bidomain approach (Biktashev et al., 1996), for a single isolated cell with an intracellular domain, $\mathcal{I}$, external domain, $\mathcal{E}$, and the membrane surface, $\mathcal{M}$, and introduce the electrostatic potential $\phi_{\mathrm{i}}$ and $\phi_{\mathrm{e}}$ in $\mathcal{I}$ and $\mathcal{E}, u_{\mathrm{i}}$ and $u_{\mathrm{e}}$ as limit values of $\phi_{\mathrm{i}}$ and $\phi_{\mathrm{e}}$ at $\mathcal{M}$, and electric charge densities $q_{i}$ and $q_{e}$ at the inside and outside surface of the membrane

$$
\begin{aligned}
& \partial_{t} q_{\mathrm{i}}=\sum_{\mathrm{i}} \Delta_{\mathrm{M}} u_{\mathrm{i}}-f(u)+\sum_{\mathrm{i}}\left(\nabla \phi_{\mathrm{i}}, \mathbf{n}\right), \\
& \partial_{t} q_{\mathrm{e}}=\sum_{\mathrm{e}} \Delta_{\mathrm{M}} u_{\mathrm{e}}+f(u)-\sum_{\mathrm{e}}\left(\nabla \phi_{\mathrm{e}}, \mathbf{n}\right),
\end{aligned}
$$

here $f$, is the transmembrane current, $\sum_{\mathrm{i}, \mathrm{e}}$ are specific conductivities, and $\Delta_{M}$ is the Laplacian operator on the membrane surface. After defining $f(\boldsymbol{r}, t), \boldsymbol{r} \in \mathcal{M}$, through local values of $u, v$ and $w$, and local kinetic equations for $v$ and $w$ from (1), these equation form a closed system, which determine evolution of the distribution of electric properties over the cell at given $\mathbf{E}(t)$, and so describe the action of the external electric field onto the cell.

We will now simplify this extensive nonlinear system of partial differential equations to a system of equations of the form:

$$
\begin{aligned}
C \partial_{t} u & =f(u, v, w)+\hat{L} u+\hat{I} \mathbf{E} \\
\partial_{t} v & =g(u, v, w) \\
\partial_{t} w & =h(u, v, w)
\end{aligned}
$$

where $u, v$ and $w$ are now functions of time and position on the membrane, $\hat{L}$ is a linear (generally, integro-differential) operator in a space of scalar functions on the membrane, and $\hat{I}$ is a linear operator mapping vectors $\mathbf{E}$ to scalar functions on the membrane. The specific forms of $\hat{L}$ and $\hat{I}$ depend on the geometry of $\mathcal{M}$ and on the coefficients $\sigma_{\mathrm{i}, \mathrm{e}}$ and $\sum_{\mathrm{i}, \mathrm{e}}$. We use the following properties of these operators:

- $\hat{L} u$ vanishes if $u$ is spatially homogeneous over the membrane,

- the integral of $\hat{L} u$ over the membrane surface is zero for any $u$, and

- the integral of $\hat{I} \mathbf{E}$ over the membrane surface is zero for any $\mathbf{E}$.

We now construct a simplified model, that retains the main features of (17). We approximate all functions $u(x), v(x)$ and $w(x)$ by piecewise constant functions, taking, at each time instant only two values at two different and fixed parts of the membrane. Denoting the two parts of the membrane by indices + and,$-(17)$ is then rewritten in the form

$$
\begin{aligned}
C \partial_{t} u_{+} & =f\left(u_{+}, v_{+}, w_{+}\right)+\alpha\left(u_{-}-u_{+}\right)+I_{\mathrm{ext}}(t) \\
C \partial_{t} u_{-} & =f\left(u_{-}, v_{-}, w_{+}\right)+\alpha\left(u_{+}-u_{-}\right)-I_{\mathrm{ext}}(t) \\
\partial_{t} v_{ \pm} & =g\left(u_{ \pm}, v_{ \pm}, w_{ \pm}\right) \\
\partial_{t} w_{ \pm} & =h\left(u_{ \pm}, v_{ \pm}, w_{ \pm}\right)
\end{aligned}
$$

where the signs in the last two equations are either all + or - .

The constant $\alpha$ is the effective conductivity of the cell in this two-compartment approximation. $I_{\mathrm{ex}}(t)$ 
is the current produced by the external source and crossing the cell.

The system (18) contains a singular small parameter, the ratio of the characteristic times of the intracellular conductivity $\alpha, \tau_{\alpha}$, and of the membrane excitability, $\tau_{\mathrm{f}}$ that can be excluded by 'adiabatic' arguments; and if the duration of pulses $I_{\text {ext }}$ is shorter than the characteristic time $\tau_{\mathrm{h}}$ of the slow variables then $w_{+} \approx w_{-} \approx W$. This final simplification gives:

$$
\begin{aligned}
C \partial_{t} U= & \frac{1}{2}\left(f\left(U+\frac{1}{2 \alpha} I_{\mathrm{ext}}(t), v_{+}, W\right)\right. \\
& \left.+f\left(U-\frac{1}{2 \alpha} I_{\mathrm{ext}}(t), v_{-}, W\right)\right) \\
\partial_{t} v_{ \pm}= & g\left(U \pm \frac{1}{2 \alpha} I_{\mathrm{ext}}(t), v_{ \pm}, W\right) \\
\partial_{t} W= & h\left(U, \frac{v_{+}+v_{-}}{2}, W\right)
\end{aligned}
$$

This model is almost as simple as the original (1) ordinary differential equation (e.g. it has three equations more than the 17 variable Oxsoft ordinary differential system we use for ventricular excitation), but describes the effect of external current. This has been obtained from (18) assuming the characteristic time of the external current pulses, $\tau_{\mathbf{l}}$, is

$$
0.1 \mathrm{~ms} \sim \tau_{\alpha} \ll \tau_{\mathrm{I}} \sim \tau_{\mathrm{f}} \sim \tau_{\mathrm{g}} \ll \tau_{\mathrm{h}} \sim 10 \mathrm{~ms} .
$$

In practice $\tau_{\mathrm{I}}, \tau_{\mathrm{f}}$ and $\tau_{\mathrm{g}}$ are all of the order of $1 \mathrm{~ms}$.
The general model (17) can be simplified by adiabatic arguments to

$$
C \partial_{\mathrm{t}} U=\int_{\mathcal{M}} f\left(U+\hat{L}^{-1} \hat{I} \mathbf{E}(t), v, W\right) \mathrm{d} \mathcal{M},
$$

with separate ordinary differential equations for $v$ at each point of the membrane. If the external field $\boldsymbol{E}$ is fixed in direction and varies only in magnitude, then the surface integral in (20) can be reduced, in a Lesbegue style, to an ordinary integral

$$
C \partial_{t} U=\int f(U+s E(t), v, W) K(s) \mathrm{d} s,
$$

where the kernel $K(s)$ is determined by the cell geometry, the conductivities, and the direction of the external field, and because of electroneutrality of the cell

$$
\int K(s) \mathrm{d} s=1 .
$$

The simple model (19) corresponds to evaluation of the integral in (21) at two points $s= \pm(1 / 2 \alpha)$.

The strength-duration curve - the threshold external current $I_{\text {ext }}$ as a function of stimulus duration for the two models (18) and (19) - coincides with a very good precision. Thus $\alpha$ is large enough for the adiabatic approximation to be valid.

The generalization of equations (19) for spatially extended tissue is straightforward as the deviation of each individual cell from an isopotential state takes

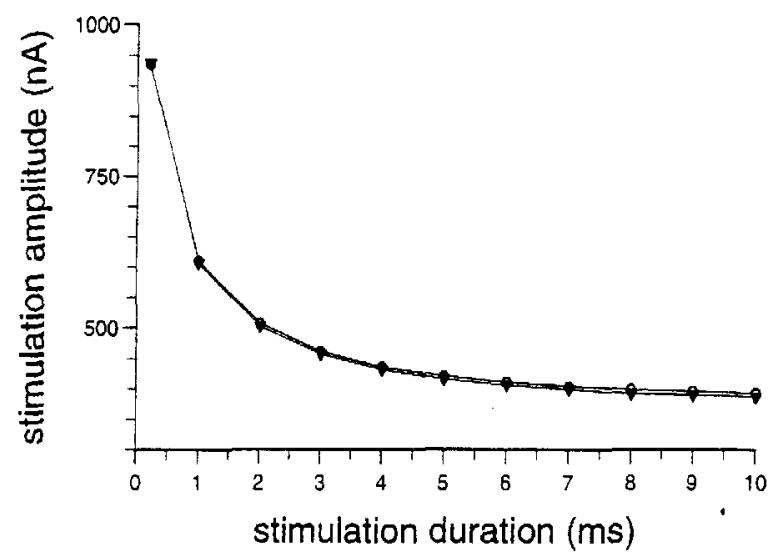

FIGURE 1.6 Excitation threshold, $\mathrm{nA}$, as a function of stimulus duration, $\mathrm{ms}$, in a single cell (open circles) and and simplified models (solid triangles) with $f(), g()$ and $h()$ were described by the Oxsoft guinea pig ventricle myocyte model that has 17 kinetic variables. $\alpha$ was $10 \mu \mathrm{S}$. 
place only during the short time periods of external stimulation, and outside these periods cable theory gives

$$
\begin{aligned}
\partial_{t} U= & \frac{1}{2 C}\left(f\left(U+\frac{1}{2 \alpha} I_{\mathrm{ext}}(t), v_{+}, W\right)\right. \\
& \left.+f\left(U-\frac{1}{2 \alpha} I_{\mathrm{ext}}(t), v_{-}, W\right)\right)+D \partial_{x}^{2} U \\
\partial_{t} v_{ \pm}= & g\left(U \pm \frac{1}{2 \alpha} I_{\mathrm{ext}}(t), v_{ \pm}, W\right) \\
\partial_{t} W= & h\left(U, \frac{v_{+}+v_{-}}{2}, W\right)
\end{aligned}
$$

where $U, v_{ \pm}$and $W$ are now functions not only of time $t$, but also of distance along the fibre $x$, and the diffusion coefficient for voltage, $D$, is proportional to the intercellular conductivity. $D=1.25 \mathrm{~cm}^{2} / \mathrm{s}$ gives a conduction velocity of $0.6 \mathrm{~m} / \mathrm{s}$ for a solitary wave through resting tissue. The value of $D$ is necessary only for the interpretation of spatial scales as equations (22) are invariant under simultaneous change of spatial scales and coefficient $D$.

We now apply this approach to evaluate the defibrillation threshold for a tissue, i.e. the amplitude of an externally applied current pulse necessary to abolish all propagating waves, and compare it with the prediction of the asymptotic theory of defibrillation for our model. This theory was described in Keener (1996) and Pumir and Krinsky (1996) and is based on separate consideration of the slow and fast processes during the process of propagation (Fife, 1976; Tyson and Keener, 1988), and assumes that on the fast time scale the medium has two alternative stable equilibria, which depend on the slow variables. The propagation of the wavefront, in the fast time scale, is a trigger wave between the equilibria that is either 'antegrade', when the excited region grows, or 'retrograde' propagation, when the excited region shrinks. The wavefront of a propagating pulse is an antegrade trigger wave, and its back a retrograde wave. In a resting medium, the upper, 'excited' equilibrium is more stable, so a suprathreshold perturbation produces an antegrade trigger wave, and the excited region expands. During the excited state, the evolution of the slow variables lowers the stability of the excited state while the stability of the resting state increases, until a retrograde trigger wave can propagate.

The boundary between these classes is in the state space of the slow variables, and corresponds to the values of these variables when the two equilibria are equally stable. If $u$ is much faster than both $v$ and $w$, this 'equal stability' is represented by a 'Maxwell (a)

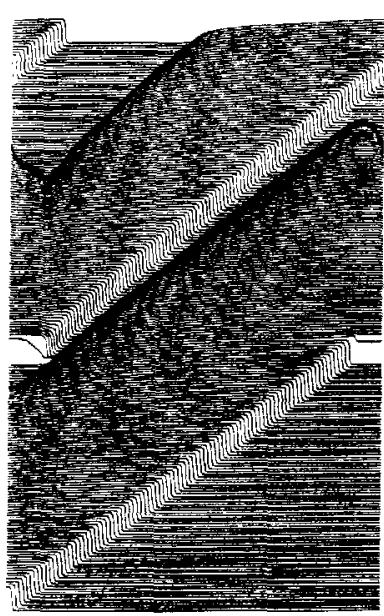

(b)

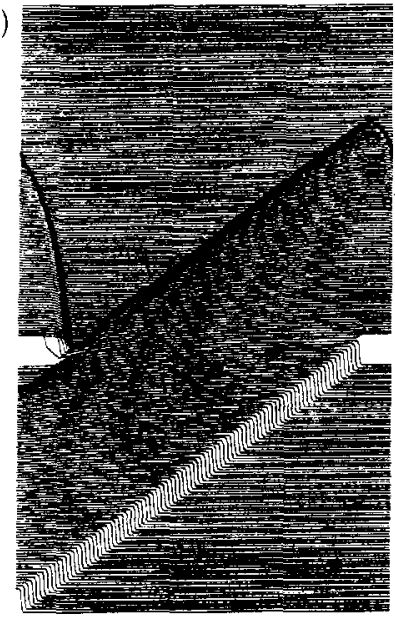

FIGURE 1.7 (a) Subthreshold (600 nA, $2 \mathrm{~ms}$ ) and (b) suprathreshold ( $800 \mathrm{nA}, 2 \mathrm{~ms}$ ) response of a re-entrant wave in one-dimensional ring to a defibribrillating shock. In both cases the wavefront is initially advanced, in the suprathreshold case the wavefront collapses back to its position at the time the pulse was applied, and meets its waveback. 

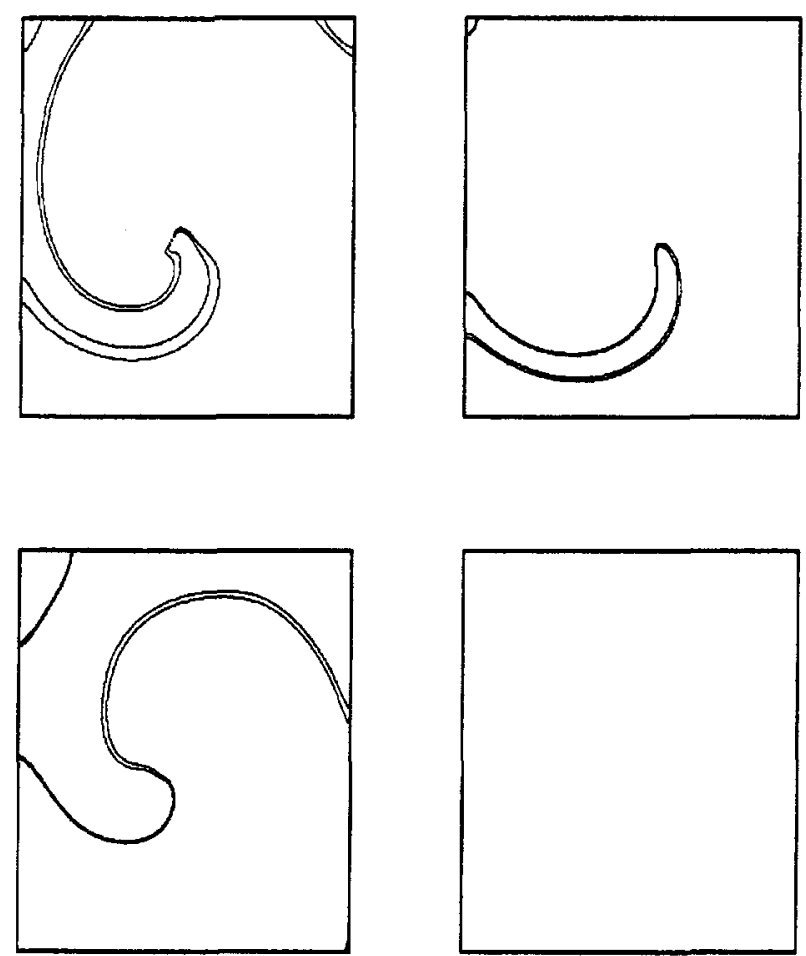

FIGURE 1.8 Wavefront and waveback of a spiral wave solution before application of the defibrillating pulse, $20 \mathrm{~ms}, 80 \mathrm{~ms}$ and $120 \mathrm{~ms}$ after application of the defibrillation pulse, when all activity is extinguished.

rule', for the right-hand side of the fast excitability equation:

$$
\int_{u_{\mathrm{resting}(v, w)}}^{u_{\mathrm{excied}(v, w)}} f(u, v, w) \mathrm{d} u=0
$$

In biophysical excitation equations the theory is applicable as long as the 'slow processes' $v$ and $w$ are slow enough for the asymptotic approach to be valid. The margin (23) between preferably-excited and preferably-resting cells is a manifold of codimension 1 , and so finding the exact defibrillation condition would imply finding the points with the highest threshold on this manifold. We do not need to search the whole space, only in its subset, corresponding to states of cells present in the tissue in the moment of defibrillation. For a spiral wave solution of the two-dimensional analogue of (22), there is a narrow gap between the waveback of the spiral wave and the following wavefront. The response of such a counter-clockwise rotating re-entrant spiral (Figure 8 ) to a brief defibrillating pulse is similar to that seen in the one-dimensional model: the wavefront is forced forward, and then relaxes back to its position at the time the shock was applied, while the waveback continues to rotate counterclockwise. All activity is extinguished when the waveback reaches the wavefront.

\section{DEFIBRILLATION BY RESONANT DRIFT}

A spiral wave can be forced to move by a spatially uniform, time periodic perturbation of appropriate frequency (Agladze et al., 1987; Davydov, et al., 1988). We have presented a phenomenological theory for such resonant drift (Biktashev and Holden, 1993). Resonant drift in the location of a spiral occurs when the frequency of perturbation is the same as the frequency of rotation of the spiral; in effect, each perturbation is applied at the same phase of rotation and so has the same effect on the spiral; since the spiral is stable this effect 
can only be a displacement in tip position and/or a change in phase of the spiral. This general property of excitable media has been exploited as a means of moving spiral waves to the boundaries of the medium. A boundary can repel a resonantly drifting spiral, and this reflection of the spiral can be overcome by appropriate timing of the repetitive perturbation, and so the spiral can be driven onto the inexcitable boundaries and be extinguished (Biktashev and Holden 1995b). In principle, resonant drift under feedback control could provide a means of eliminating re-entrant activity in cardiac tissue by using small amplitude, repetitive electrical stimulation (Biktashev and Holden 1994). This will be practical only if any re-entry is eliminated within a reasonable time, say less than $30 \mathrm{~s}$.

We have used resonant drift to move and control re-entrant waves in simplified FitzHugh-Nagumo models of excitable media, and we have shown that using feedback control of the stimulation can provide elimination of the re-entrant activity, even when the period of re-entry is unknown or is varying in time, or in presence of inhomogeneities, or when there are multiple re-entrant sources present (Biktashev and Holden 1993, 4, 5a). Defibrillation by resonant drift under feedback control for the ventricle CML model is illustrated in Figure 9.

These computations have been extended to atrial and ventricular models (Biktashev and Holden 1995b, 6). The effect of strictly periodic repetitive stimulation is to produce a drift of the position of the spiral, and general theory (Davydov et al., 1988; Biktashev and Holden, 1995) predicts that this drift would be along a circle, with a drift velocity that depends upon stimulation amplitude and an angular velocity equal to the difference between the stimulation frequency and the resonant frequency of the spiral, where the latter also depends on stimulation amplitude and is close to the frequency of free spiral rotation when the amplitude of stimulation is small. Such an induced circular drift is illustrated in Figure 10, so the behaviour of the atrial tissue model is still consistent with the general theory, even though the theory was developed for a medium without refractoriness (Davydov et al., 1988), and for a medium with a refractory period but using linear perturbation theory (Biktashev and Holden, 1995a) which is not formally applicable to the atrial tissue model because of the steepness of the propagation front.

Strictly periodic stimulation is not very effective in extinguishing the spiral, as it is difficult to choose appropriate conditions that would cause the spiral wave to reach the inexcitable boundaries. Drift in a straight line towards the boundary is produced only in the case of an exact resonance, and even in this case the drift may not cause annihilation of the spiral, as the resonantly drifting spiral can be reflected by the boundary (Biktashev and Holden 1993).

However, feedback controlled stimulation is more effective at driving the spiral to the boundaries (Biktashev and Holden 1994). The feedback dynamically adjusts the frequency of stimulation to the instantaneous frequency of rotation, thus damping the de-tuning of resonance caused by changes in the frequency of rotation as the resonantly drifting spiral

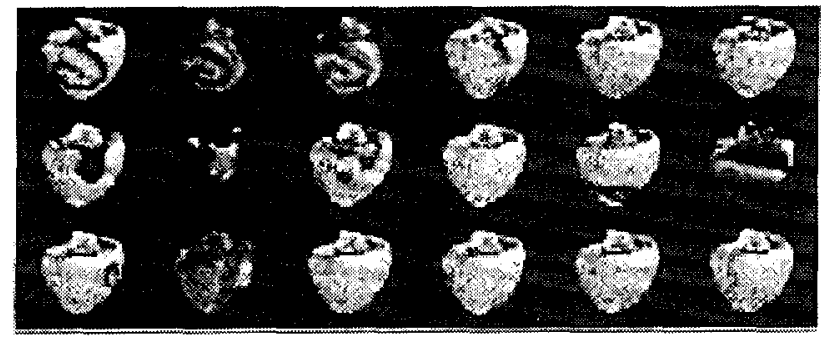

FIGURE 1.9 Frames from a movie illustrating elimination of re-entrant activity in canine ventricle CML model by spatially uniform, low amplitude stimulation triggered by the arrival of a wavefront at a point on the right epicardial surface. The first few stimuli leave re-entrant sources deep in the heart, so activity re-emerge on the surface. However, finally (within a few seconds of canine time) all re-entrant activity is eliminated. 


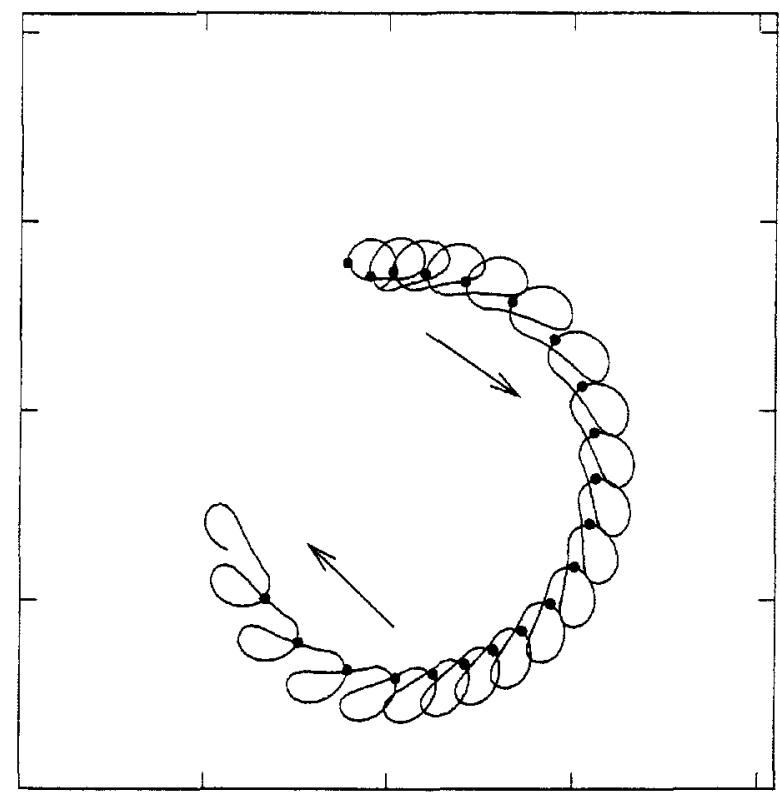

FIGURE 1.10 Effect of strictly periodic repetitive stimulation with period $77.2 \mathrm{~ms}$ and amplitude $4 \mathrm{mV} / \mathrm{ms}$ on the trajectory of the tip of the spiral (continuous curve) for the rabbit atrial tissue model, and the phase $(\bullet$ ) of the spiral, given by the position of the tip at instants of each stimulus. The averaged trajectory of the tip is nearly circular. The angular velocity of the averaged drift is the difference between the vortex frequency and the stimulation frequency.

approaches a boundary, that leads to reflection of resonantly drifting spirals.

Repetitive stimulation, triggered each time the wavefront reaches a recording site produces a drift of the spiral, which in the standard atrial tissue model always drives the spiral onto the boundary (Figure 11). In FitzHugh-Nagumo models, the interaction between the resonantly drifting spiral and the boundary was strong enough and the drifting spiral could stop near the boundary and persist, if the simulation amplitude was below a certain threshold. In the atrial tissue model, the interaction between the spiral and the boundary is weak and the annihilation was always produced, even at lowest stimulation amplitudes used $(0.5 \mathrm{mV} / \mathrm{ms})$.
In the ventricular tissue model, that shows a complicated pattern of meander, changing from a linear to a multi-lobed core, resonant drift can still be use to drive the meandering spiral onto the medium boundaries (Figure 12) (Biktashev and Holden, 1996). The repetitive stimuli used in the above computations are all much less than the threshold for a single 'defibrillating shock' of the same duration, i.e. the minimal shock which is sufficient for suppressing the spiral.

The surprising results of these computations are that, in spite of the strong non-linearities of the excitation equations, the main qualitative predictions of the phenomenological theory for feedback driven resonant drift developed in Biktashev and Holden

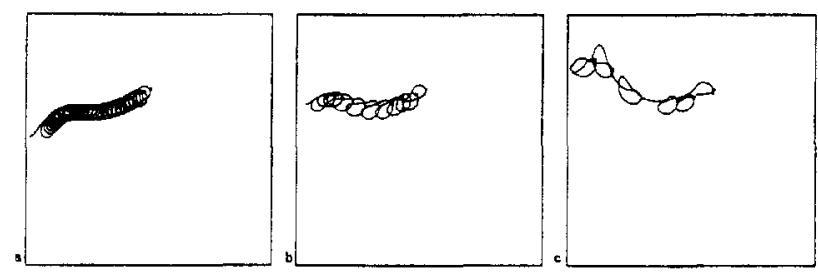

FIGURE 1.11 Tip trajectories under feedback controlled, resonant driving of atrial tissue model. 

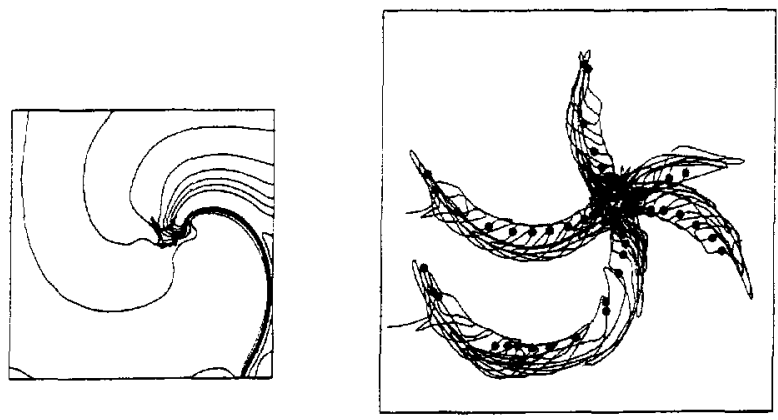

FIGURE 1.12 (Left) Contours of re-entrant wave moving around an irregular, multi-lobed core and (Right) tip trajectories under feedback controlled, resonant driving of ventricular tissue model. When the wavefront of the spiral wave (depolarisation through $-10 \mathrm{mV}$ ) reached a recording site in the bottom left hand corner, a $2 \mathrm{~ms}, 4 \mathrm{~V} / \mathrm{s}$ depolarising perturbation was added after a fixed delay. Each trajectory is for a different delay, from 0 to $100 \mathrm{~ms}$, and corresponds to applying the perturbation at a different phase of the spiral.

(1994) have proved to be valid (except for linear dependence upon stimulation amplitude), and that even with stimulus sizes $10 \%$ of the single shock defibrillation threshold drift velocities of $1 \mathrm{~cm} \mathrm{~s}^{1}$ are reached. Despite all the simplifications made, at least the order of magnitude of this velocity should be realistic. Thus we anticipate that resonant drift produced by low amplitude, spatially uniform repetitive stimulation under feedback control can extinguish re-entrant arrhythmias in mammalian atrial tissue within about $10 \mathrm{~s}$, and so can provide a practical technique for extinguishing atrial flutter.

\section{CURRENT PROBLEMS}

In spite of its practical importance, the processes of defibrillation remain obscure. Most theoretical approaches have been based on linear models (Sepulveda et al., 1989; Krassowska et al., 1990, Knisley et al., 1994) while some numerics of nonlinear models (Cartee and Plonsey, 1992) and theoretical studies with simplified models have been attempted (Pumir and Krinsky, 1996). The reason for this lack of progress is the combination of nonlinearity with the necessity for a representation of the complicated spatial structure for every cell. We have overcome these problems by applying a series of well known methods, to fulfill nonlinear averaging; the result is the simplified models of (18) and (22). This reduction of an infinite dimensional system to an ordinary differential system may be of value in a range of applications of nonlinear science.

These simplified models have been verified numerically and allow us to use biophysically detailed excitation equations, and so we are now in a position to provide a quantitative, theoretical explanation for the effects of changes in parameters in the excitation equations on the defibrillation threshold, and to design optimal defibrillation pulse parameters. Experimental techniques now exist (Zhou et al., 1995) for testing such quantitative descriptions of the mechanisms of defibrillation.

\section{Acknowledgement}

The work described here was done in collaboration with Dr Biktashev and supported by grants from the Wellcome Trust $(042352,044365)$ and the EPSRC ANM initiative (GR/J35641), and was written on a Lever Gulme Trust Fellowship.

\section{References}

Agladze, K. I., Davydov, V. A. and Mikhailov, A. S. (1987) An observation of resonance of spiral waves in distributed excitable medium. Pis'ma v ZETP, (in Russian, see English translation in Sov. Phys.-JETP Letters), 45, 601-603.

Biktashev, V. N. and Holden, A. V. (1993) Resonant drift of an autowave vortex in a bounded medium. Physics Lett. A, 181, 216-224.

Biktashev, V. N. and Holden, A. V. (1994) Design principles for a low voltage cardiac defibrillator based on the effect of feedback resonant drift. J. Theor. Biol, 169, 101-112. 
Biktashev, V. N. and Holden, A. V. (1995a) Resonant drift of autowave vortices in $2 \mathrm{D}$ and the effects of boundaries and inhomogeneities., Chaos Solitons and Fractals, 5, 575-622.

Biktashev, V. N. and Holden, A. V. (1995b) Control of reentrant activity in a model of mammalian cardiac tissue., Proc. Roy. Soc. Lond. B, 260, 211-217.

Biktashev, V. N. and Holden, A. V. (1996) Re-entrant activity and its control by resonant drift in a two-dimensional model of isotropic homogeneous ventricular tissue,, Proc. Roy. Soc. $B, 263,1373-1382$.

Biktashev, V. N., Holden, A. V. and Zhang, H. (1997) A model for the action of external current onto excitable tissue., International Journal of Biturcation and Chaos, $17(2)$, in press.

Cartee, L. A. and Plonsey, R. (1992) Active response of a onedimensional cardiac model with gap junctions to extracellular stimulation. Medical and Biological Engineering and Computing, 30, 389-398.

Davydov, V. A., Zykov, V. S., Mikhailov, A. S. and Brazhnik, P. K. (1988) Drift and resonance of spiral waves in distributed media, $I z v$. Vuzov-Radiofizika, (in Russian, see English translation in Sov. Phys.-Radiophysics., 31, 574-582.

Fife, P. C. (1976) Boundary and interior transition layer phenomena for paris of second-order differential equations, J. Math. Anal. Appl., 54, 497-521.

Holden, A. V., Poole, M. J. and Tucker, J. V. (1996) An algorithmic model of the mammalian heart: propagation, vulnerability, re-entry and fibrillation, International Journal of Bifurcation and Chaos, 6, 1623-1635.

Holden, A. V. and Zhang, H. (1995) Characteristics of atrial reentry and meander computed from a model of a rabbit single atrial cell., $J$. Theoretical biology, 175, 545-551.

Keener, J. P. (1996) Direct activation and defibrillation of cardiac tissue., J. Theoretical biology, 178, 313-324.
Noble, D. (1994). Oxsoft HEART version 3.8 manual, (Oxsoft, Oxford).

Knisley, S. B., Hill, B. and Ideker, R. E. (1994) Virtual electrode effects in myocardial fibres, Biophys. J., 66, 719-728.

Krassowska, W., Frazier, D. W., Pilkington, T. C. and Ideker, R. E. (1990) Potential distribution in three-dimensional anisotropic medium. IEEE Biomed. Eng., 37, 267-284.

Panfilov, A. V, and Holden, A. V. (eds) (1997) The Computational Biology of the Heart, (J. Wiley, Chichester).

Plonsey, R. and Barr, R. C. (1996) Effect of microscopic and macroscopic discontinuities on the response of cardiac tissue to defibrillating (stimulating) currents., Medical and Biological Engineering and Computing, 24, 130-136.

Pumir, A. and Krinsky, V. (1996) How does an electric current defibrillate cardiac muscle? Physica D, 91, 205-219.

Sepulvedâ, N. G., Roth, B. J. and Wikswo Jr, J. P (1989) Current injection into a two-dimensional anisotropic medium, Biophys. J., 55, 987-999.

Tyson, J. J. and Keener, J. P. (1998) Singular perturbation theory of traveling waves in excitable media (a review), Physica $D$, 32, 327-361.

Winslow, R. L., Varghese, A., Noble, D., Adlakha, C. and Hoythya, A. (1993) Generation and propagation of ectopic beats induced by spatially localised $\mathrm{Na}-\mathrm{K}$ pump inhibition in atrial network models. Proc. Rov. Soc. Lond. B, 254, 55-61.

Zipes, D. P. and Jalife, J. (eds) (1995) Cardiac Electrophysiology - From Cell to Bedside, (W. B. Saunders, Philadelphia).

Zhou, X., Ideker, R. E., Blitchington, T. F., Smith, W. M. and Knisley, S. B. (1995) Optical transmembrane potential measurements during defibrillation-strength shocks in perfused rabbit hearts., Circulation Research, 77, 593-602. 


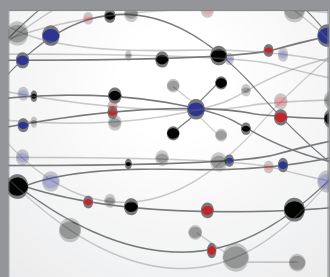

The Scientific World Journal
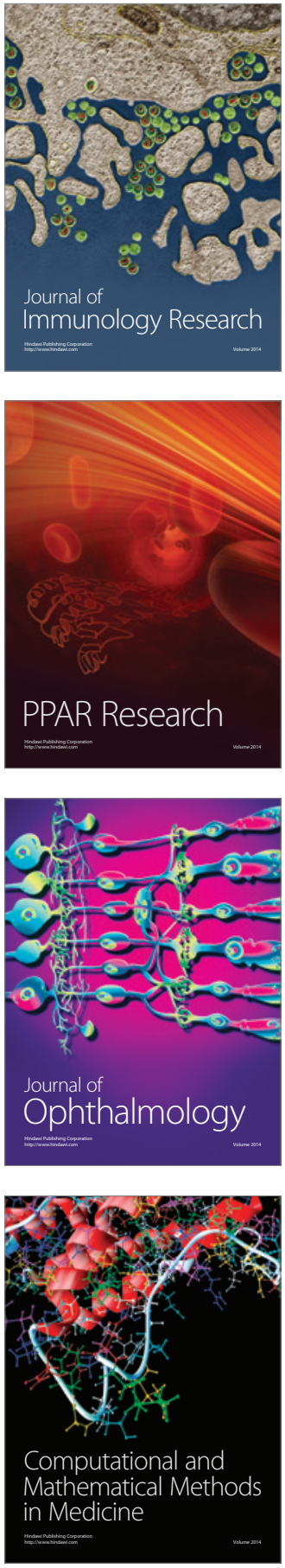

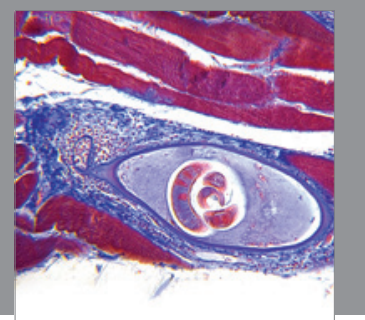

Gastroenterology

Research and Practice
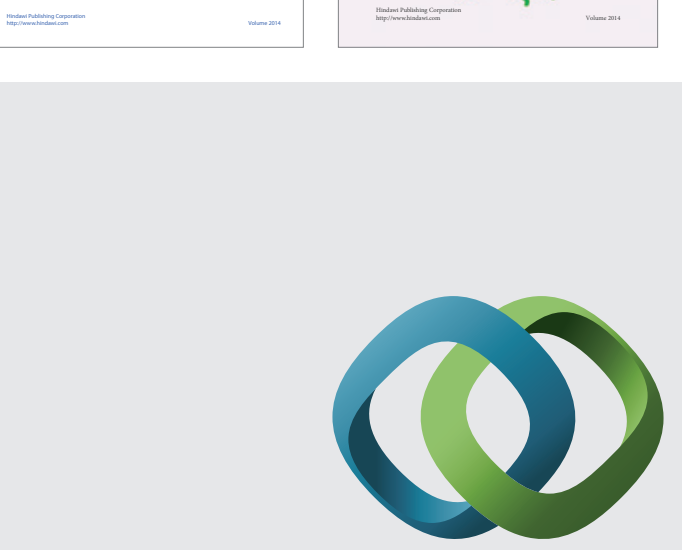

\section{Hindawi}

Submit your manuscripts at

http://www.hindawi.com
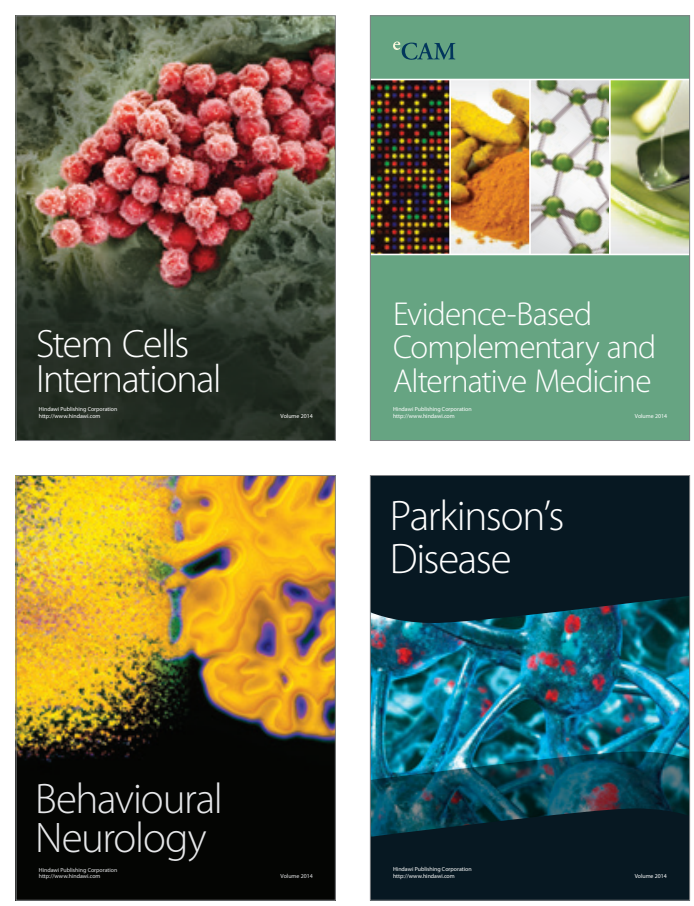

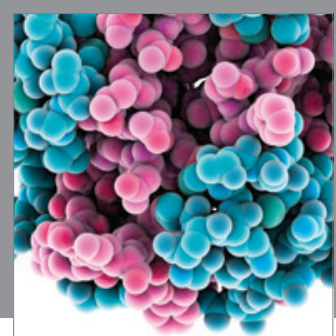

Journal of
Diabetes Research

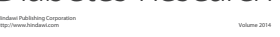

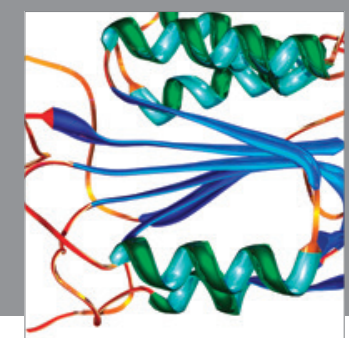

Disease Markers
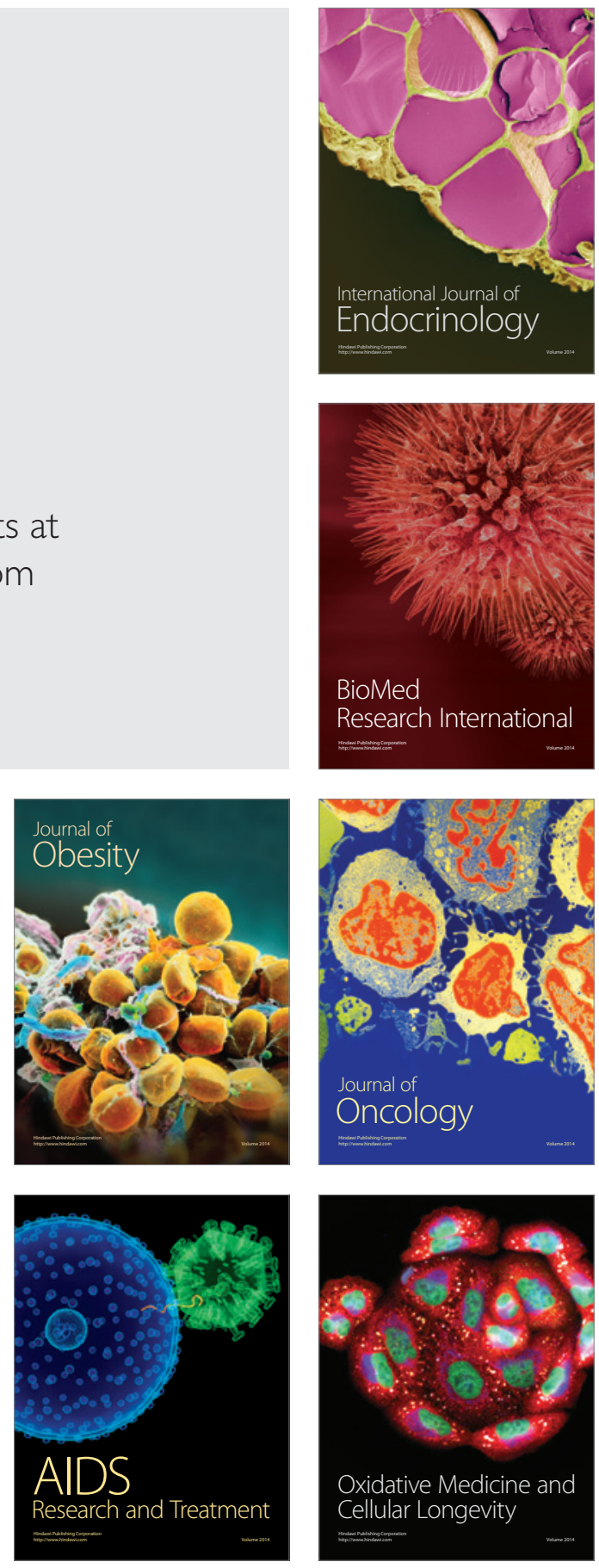